\title{
Variational Segmentation Using Dynamical Models for Rigid Motion
}

\author{
Jan Erik Solem and Anders Heyden \\ Applied Mathematics Group \\ School of Technology and Society \\ Malmö University, Sweden \\ \{jes, heyden\}@ts.mah.se
}

\begin{abstract}
This paper deals with the segmentation of multiple moving objects in image sequences. A method for estimating the motion of objects without the use of features is presented. This is used to predict the position and orientation in future frames of the sequence. Experiments on real data show that this estimation can be used to improve segmentation.
\end{abstract}

\section{Introduction}

Segmentation is the process of decomposing an image in meaningful regions, such as e.g. different objects and background. In this paper, segmentation in image sequences of objects undergoing rigid (and possibly non-rigid) motion is studied. Based on the segmentation in previous frames we are interested in obtaining estimates of the object position in the next frame. We are especially interested in regions and boundaries that are difficult to track using standard methods. Examples are textureless objects and objects having boundaries without easily identifiable points. If the scene contains textured regions where feature points can be detected and tracked through the sequence, the estimation of a rigid (or non-rigid) transformation between frames is straightforward. When the objects are featureless however, it is much harder.

A method for estimating the between-frame translation and rotation directly from the object boundaries without the use of features or landmarks is presented. Based on these estimates, dynamic models of the motion of the scene objects can be derived to estimate the position in new frames. This gives a useful online initialization algorithm. We use the Chan-Vese segmentation model [1 and a simple linear motion model to illustrate the procedure for a number of examples. The procedure is general enough to be used as initialization for curve based methods such as traditional active contour methods in the form of snakes [2], geometric active contours [3], and other similar models [4,5].

We will use a level set representation 667] for representing the image regions and the boundary curve of the segmentation. The proposed estimation method uses normal velocities and is therefore compatible with any representation of the boundary, explicit or implicit, cf. 8]. 
The method in itself is based on the property that any curve evolution can be partitioned into translation, rotation and deformation. This was earlier studied in [9] and [10]. In this paper we use the partition of a particular flow and integrate over the evolution time to find the rigid component of the object displacement in the image.

When segmenting space-time data, a simple (and effective) idea is to perform the segmentation in the space-time volume using surfaces. This was studied in e.g. 11]. This enables regularization of the segmentation both within images and between images. However, a necessary requirement is that the image sequence is dense enough to make the between image difference sufficiently small. Large displacements are clearly not handled using such volumetric methods. Another (perhaps less serious drawback is that volumetric techniques are "global" since the entire sequence is used and the method can not be used to estimate object positions at consecutive frames.

The paper is organized as follows; Section 2 presents the necessary background material, Section 3 shows how translation and rotation can be estimated based on the shape of the objects in consecutive frames, Section 4 describes how this can be used to build motion models, Section 5 presents some experiments and Section [6] our conclusions.

\section{Background}

As a courtesy to the reader, the necessary background on the level set method, geometric gradient descent and on determining rotation and translation by orthogonal projections is briefly recalled here.

\subsection{The Level Set Representation}

The level set method for evolving implicit surfaces was introduced independently by $[12$ and 6 . The time-dependent curve $\Gamma(t)$ is represented implicitly as the zero level set of a function $\phi(\mathbf{x}, t): \mathbf{R}^{2} \times \mathbf{R} \rightarrow \mathbf{R}$ as

$$
\Gamma(t)=\{\mathbf{x} ; \phi(\mathbf{x}, t)=0\} .
$$

The sets $\Omega_{0}=\{\mathbf{x} ; \phi(\mathbf{x}, t)<0\}$ and $\Omega_{1}=\{\mathbf{x} ; \phi(\mathbf{x}, t)>0\}$ are called the interior and the exterior of $\Gamma$, respectively. Using this definition the outward unit normal $\mathbf{n}$ and the mean curvature $\kappa$ are given as

$$
\mathbf{n}=\frac{\nabla \phi}{|\nabla \phi|} \quad \text { and } \quad \kappa=\nabla \cdot \frac{\nabla \phi}{|\nabla \phi|} .
$$

To evolve the surface according to some derived normal velocity $v$, a PDE of the form

$$
\frac{\partial \phi}{\partial t}+v|\nabla \phi|=0
$$

is solved. For a more thorough treatment of the level set method and implicit representations, cf. [7]. 
In the variational level set method, functionals are used to derive gradient descent motion PDEs of the form (3). This is done through the use of the differential (Gâteaux derivative). The differential $d E$ is related to the $L^{2}$-gradient $\nabla E$ of a functional $E$ as

$$
d E(\Gamma) v=\langle\nabla E, v\rangle_{\Gamma}:=\int_{\Gamma} \nabla E v d \sigma
$$

where $v$ is the normal component of a perturbation of $\Gamma$, and $d \sigma$ is the curve length element. For details cf. e.g. 8 8913 . The gradient descent flow for $E$ is then obtained by solving the following initial value problem

$$
\frac{\partial \phi}{\partial t}=\nabla E|\nabla \phi|, \quad \phi(\mathbf{x}, 0)=\phi_{0}(\mathbf{x})
$$

where $\phi_{0}$ is a level set function for the initial curve $\Gamma_{0}$, specified by the user.

\subsection{The Chan-Vese Model}

Let us briefly recall the Chan-Vese model [1] which we will use to segment images in the experiments in Section 5, Let $I=I(\mathbf{x}): D \rightarrow \mathbf{R}$ denote the image to be segmented, $D \subset \mathbf{R}^{2}$ being the image domain. Also, let $\Gamma$ denote a simple closed curve in the image domain (or a non-overlapping union of such curves, bearing in mind that this is allowed in the level set framework). Consider the functional

$$
E(\boldsymbol{\mu}, \Gamma)=\frac{1}{2} \int_{\Omega_{0}}\left|I(\mathbf{x})-\mu_{0}\right|^{2} d \mathbf{x}+\frac{1}{2} \int_{\Omega_{1}}\left|I(\mathbf{x})-\mu_{1}\right|^{2} d \mathbf{x}+\alpha|\Gamma|,
$$

where $\boldsymbol{\mu}=\left(\mu_{0}, \mu_{1}\right) \in \mathbf{R}^{2}$ is a pair of parameters, $|\Gamma|$ denotes the length of the curve $\Gamma$, and $\alpha>0$ is a fixed weight. The idea of the method presented in [1] is to find a curve $\Gamma^{*}$ and a pair of parameters $\boldsymbol{\mu}^{*}$ which solves the optimization problem,

$$
E\left(\boldsymbol{\mu}^{*}, \Gamma^{*}\right)=\min _{\boldsymbol{\mu}, \Gamma} E(\boldsymbol{\mu}, \Gamma)
$$

The segmentation of the image $I$ is defined as the partition of the image domain induced by the optimal curve $\Gamma^{*}$. This partition is found using gradient descent on $\Gamma$ where the gradient is

$$
\nabla E=\frac{1}{2}\left(I(\mathbf{x})-\mu_{0}\right)^{2}-\frac{1}{2}\left(I(\mathbf{x})-\mu_{1}\right)^{2}+\alpha \kappa .
$$

It is easy to find the optimal parameters for each fixed $\Gamma$; they are simply the mean intensities of the image taken over each of the sub-domains cut out by $\Gamma$

$$
\mu_{i}(\Gamma)=\frac{1}{\left|\Omega_{i}\right|} \int_{\Omega_{i}} I(\mathbf{x}) d \mathbf{x}, \quad(i=0,1)
$$

where $\left|\Omega_{i}\right|$ denotes the area of the set $\Omega_{i} \subset \mathbf{R}^{2}$. 


\subsection{Decomposition of Evolutions}

Given a curve evolution, described by the normal velocity as in (3) or (5), it is possible to decompose this evolution into translation, rotation and deformation, cf. 9[10. In this section we briefly describe this process.

The $L^{2}$-gradient $\nabla E$, can be divided into three components $\Pi_{T} \nabla E, \Pi_{R} \nabla E$, and $\Pi_{D} \nabla E$. The two first terms are the orthogonal projections of $\nabla E$ onto the subspaces (of normal velocities at $\Gamma$ ) generated by translations and rotations, respectively, and the last term is the residual $\Pi_{D} \nabla E=\nabla E-\Pi_{T} \nabla E-\Pi_{R} \nabla E$. The residual corresponds to what is left after a rigid transformation of the curve, i.e. deformation.

The operator $\Pi_{T}$ is the projection on the subspace $L_{T}$ of $L^{2}(\Gamma)$ spanned by all translations. The elements of $L_{T}$ are then exactly the normal velocities which come from pure translation motions. The operator can be shown to be

$$
\Pi_{T} v=\mathbf{n}^{T} \mathbf{v}=\mathbf{n}^{T}\left[\int_{\Gamma} \mathbf{n n}^{T} d \sigma\right]^{-1} \int_{\Gamma} v \mathbf{n} d \sigma,
$$

for all normal velocities $v \in L^{2}(\Gamma)$, cf. [10. The positive semi-definite matrix $\int_{\Gamma} \mathbf{n n}^{T} d \sigma$, in the right-hand side, is called the structure tensor of the curve.

Let us define $\hat{\mathbf{x}}=\left[\begin{array}{cc}0 & -1 \\ 1 & 0\end{array}\right] \mathbf{x}$ as the $\pi / 2$-rotation of the vector $\mathbf{x}$. The projection on the space of rotations $L_{R}$ around a point $\mathbf{x}_{0} \in \mathbf{R}^{2}$, can be shown to be

$$
\Pi_{R} v=\frac{\mathbf{n}^{T}\left(\hat{\mathbf{x}}-\hat{\mathbf{x}}_{0}\right) \int_{\Gamma} v \mathbf{n}^{T}\left(\hat{\mathbf{x}}-\hat{\mathbf{x}}_{0}\right) d \sigma}{\int_{\Gamma}\left|\mathbf{n}^{T}\left(\hat{\mathbf{x}}-\hat{\mathbf{x}}_{0}\right)\right|^{2} d \sigma} .
$$

The point $\mathbf{x}_{0}$ in (11) is chosen such that the two subspaces $L_{T}$ and $L_{R}$ are orthogonal which means that $\mathbf{x}_{0}$ must satisfy the following vector relation $\int_{\Gamma}\left[\mathbf{n}^{T}\left(\hat{\mathbf{x}}-\hat{\mathbf{x}}_{0}\right)\right] \mathbf{n} d \sigma=0$, hence

$$
\hat{\mathbf{x}}_{0}=\left[\int_{\Gamma} \mathbf{n} \mathbf{n}^{T} d \sigma\right]^{-1} \int_{\Gamma}\left(\mathbf{n}^{T} \hat{\mathbf{x}}\right) \mathbf{n} d \sigma
$$

where, interestingly enough, the structure tensor for $\Gamma$ appears again. Since $L_{T}$ and $L_{R}$ are now orthogonal, it follows that the residual operator is also an orthogonal projection. For more details we refer to [10].

\section{Computing Translation and Rotation}

In this section we propose a method of computing translation and rotation between frames in an image sequence without the use of landmarks or features. If the boundary curve of the segmentation is represented using landmarks of some kind it is trivial to compute the translation and rotation given two curves. Since it is often difficult to reliably extract landmarks along the boundary we 
would like to do this directly in the implicit framework. The translation can be estimated using the centroid of the regions but the rotation component is more difficult. One choice is to use moments of the regions. This fails, however, when there are no dominant directions.

We propose to rigidly align the boundary curve $\Gamma_{i-1}$ from one frame to the boundary curve $\Gamma_{i}$ in the consecutive frame using the gradient descent of the area of symmetric difference functional, cf. [14,

$$
E(\Gamma)=\frac{1}{2} \int\left(\chi_{\Omega_{i-1}}-\chi_{\Omega_{i}}\right)^{2} d \mathbf{x}
$$

Here $\chi_{\Omega_{i-1}}$ and $\chi_{\Omega_{i}}$ are characteristic functions for the regions. The corresponding gradient flow for $\Gamma_{i-1}$ is given by

$$
\nabla E(\Gamma)=\frac{1}{2}-\chi_{\Omega_{i}}
$$

This evolution is projected on the space of rigid transformations using the operators in Section 2.3. After discarding the deformation part, the evolution is simply

$$
\frac{\partial \phi}{\partial t}=\left(\Pi_{T} \nabla E+\Pi_{R} \nabla E\right)|\nabla \phi|
$$

At each iteration the translation vector $\mathbf{v}$ can be recovered using the relation

$$
\mathbf{v}=\left[\int_{\Gamma} \mathbf{n n}^{T} d \sigma\right]^{-1} \int_{\Gamma} v \mathbf{n} d \sigma
$$

from (10). Similarly, the rotation angle $\omega$ is given by

$$
\omega=\frac{\int_{\Gamma} v \mathbf{n}^{T}\left(\hat{\mathbf{x}}-\hat{\mathbf{x}}_{0}\right) d \sigma}{\int_{\Gamma}\left|\mathbf{n}^{T}\left(\hat{\mathbf{x}}-\hat{\mathbf{x}}_{0}\right)\right|^{2} d \sigma} .
$$

Now we just sum up the angles and displacements for all iterations, multiplying with the length of the time step of the rigid alignment evolution, to obtain the rotation $\omega_{i}$ and translation $\mathbf{v}_{i}$ of the object between the two images. In the next section we will use this to estimate the future values $\omega_{i+1}$ and $\mathbf{v}_{i+1}$.

\section{Initialization Using Motion Models}

In this section we will use the rigid body transformations obtained from the procedure above to initialize the segmentation in a given image. The goal is to estimate the position and orientation of the objects in frame $i+1$ given the observations in previous frames $1, \ldots, i$. If the images are time-stamped one can fit polynomials, splines or curves to the previous values of $\mathbf{v}$ and $\omega$ and extrapolate to predict the values that transform the object to the next frame. 
Here, for the sake of simplicity, we will use a simple linear model which states that $\mathbf{v}_{i+1}=\mathbf{v}_{i}$ and $\omega_{i+1}=\omega_{i}$. We consider the extension to other more sophisticated models to be an interesting field for future studies. Examples of interesting extensions of the simple model are:

- Periodic motion. Many medical examples, such as segmentation in cross sections of MR cardiac images, show periodic behavior in the sequence.

- Physical models. Incorporate (angular)velocity, acceleration and (angular)momentum of the objects.

- Missing data. Interpolate position and orientation between frames, in missing frames or when the objects are occluded.

Note that the objects in the scene are treated as a single rigid constellation if they are represented using one level set function. If there are several independent rigid parts moving non-rigidly one function for each part can be used. In this case the translation and rotation is estimated for each function at each iteration.

\section{Experiments}

The framework presented above is applied to some examples in this section. We show three examples of moving scenes captured by a digital camera. The scenes contain objects with very little texture and feature points that can be tracked. We show that the proposed method of estimating rigid motion and using this to predict the position of the objects in future frames makes it possible to reliably segment the objects.

In all three examples, the initial segmentation is obtained using standard level set implementation of the Chan-Vese model described in Section 2.2 and [1. This means that the boundary curve is evolved using (8) in a gradient descent,

$$
\frac{\partial \phi}{\partial t}=\left[\frac{1}{2}\left(I(\mathbf{x})-\mu_{0}\right)^{2}-\frac{1}{2}\left(I(\mathbf{x})-\mu_{1}\right)^{2}+\alpha \kappa\right]|\nabla \phi|,
$$

until steady state, starting from some initial curve. The boundary curve of the segmentation is represented implicitly and no points, landmarks or features are used. We are also assuming that the objects are moving rigidly when the motion is estimated and they are therefore represented using a single level set function.

In general, we find that the translation vectors obtained using our approach are very close to the translation obtained through the difference of the region centroids. The estimation of the angles appear, at least qualitatively, to be accurate.

The first sequence consists of six images of three apples in a translation-like motion. The images and the resulting segmentation are shown in Figure 1 from left to right and top down. The first two images are automatically segmented and the translation and rotation of the apples computed by solving (15) and integrating the translation vector and angle as described in Section 4 The white curve shows the initial curve obtained using a simple linear model for the motion. The 

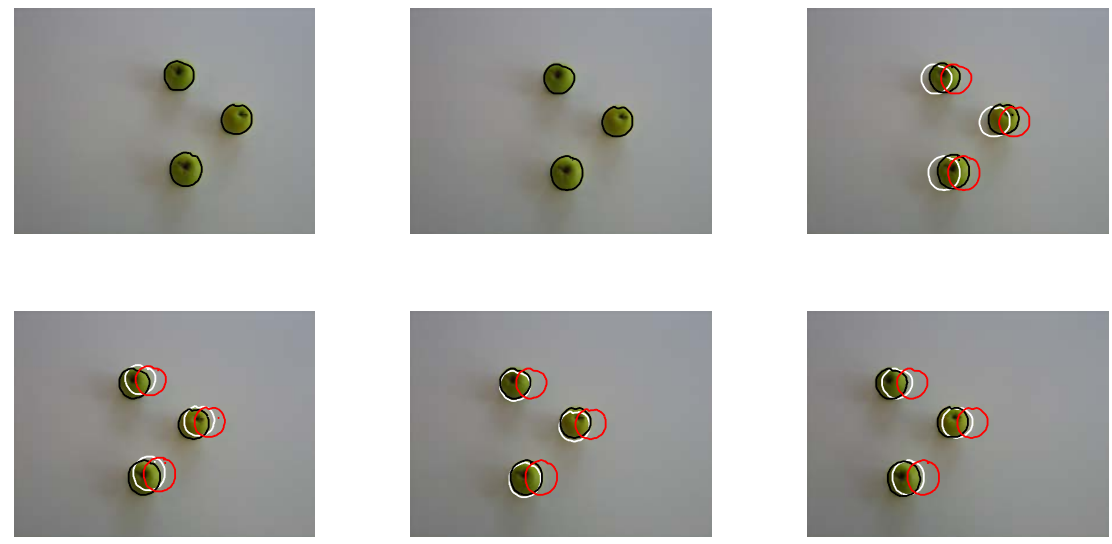

Fig. 1. Example of a translating scene (right and down). The black curve is the boundary contour of the final segmentation for each image. The white curve is the estimated position used as initial curve based on the last two frames and the red curve is the curve from the previous frame. As can be seen from the position of these curves the estimated position is better.
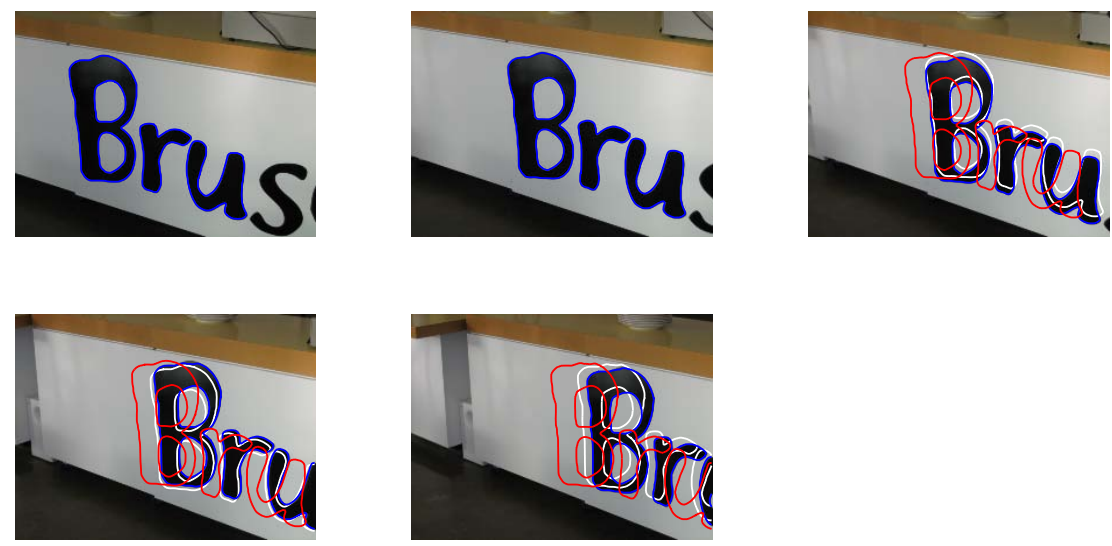

Fig. 2. Example of a translating scene (right and down). The blue curve is the boundary contour of the final segmentation for each image. The white curve is the estimated position used as initial curve based on the last two frames and the red curve is the curve from the previous frame. As can be seen from the position of these curves the estimated position is better.

red curve is the initial curve given by the boundary from the previous frame. It is clear that the initial segmentation is better with the motion model. In two of the frames the red curve is to far from the objects and the Chan-Vese motion then segments the background as the interior region with respect to the boundary. 

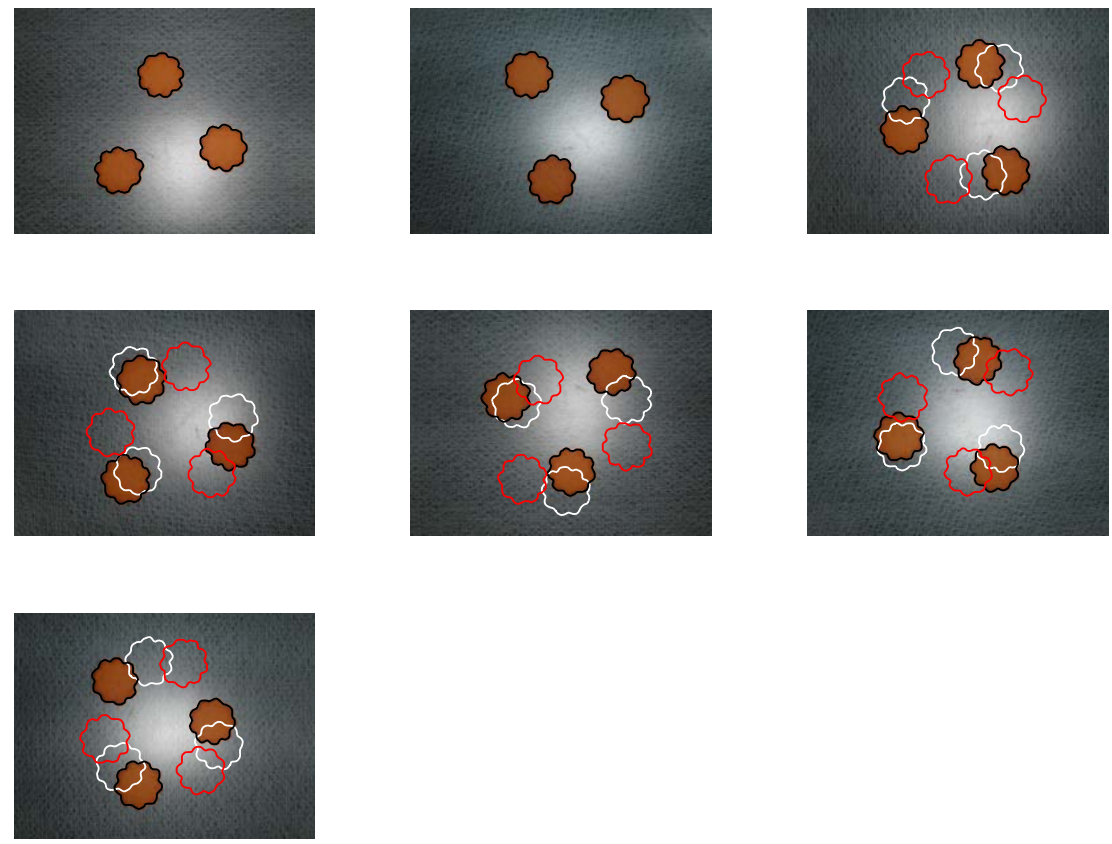

Fig. 3. Example of a rotating scene (right and down). The black curve is the boundary contour of the final segmentation for each image. The white curve is the estimated position used as initial curve based on the last two frames and the red curve is the curve from the previous frame. As can be seen from the position of these curves there are several frames where segmentation starting from the red curve would fail.

The final segmentation will of course be correct for these simple images but it takes very long time for the segmentation to converge in this case.

The second sequence shows five images of printed text on a table side. Three letters are segmented in the first and second image and the final boundary is shown in blue in Figure 2. The white curve shows the initial curve obtained using the same simple motion model as above. The red curve is the initial curve given by the boundary from the previous frame. As with the example above, it is clear that the initial segmentation is better with the motion model. The initial red curve in the last frame is too far from the letters and as a consequence the segmentation is slow and "inverted".

The third example is a sequence containing seven images of three cookies on a table undergoing a rotation-like motion. The images and the resulting segmentation is shown in Figure 3. The black curve is the boundary contour of the final segmentation for each image. The white curve is the estimated position used as initial curve based on the last two frames and the red curve is the curve from the previous frame. The in-plane rotation of the cookies is quite large between frames and there is no overlap of the regions enclosed by the red curves. The red curves are therefore a very bad initialization. A "standard" ring-like pattern, 
cf. [1], covering the image would be better in this case. The white curves however, are much better even if just a linear motion model is used.

\section{Conclusions}

This paper presented a method for estimating the motion of objects between images which can be used for featureless objects. Rotation and translation are obtained by projecting a particular warping flow on the subspace of rigid transformations and integrating over the evolution time of the flow. Using the transformation from previous frames in an image sequence makes it possible to estimate the position and orientation of the objects in future frames. Experiments show that this can be used for initialization for segmentation.

Future work and extensions include:

- More sophisticated models for the motion of the objects.

- Multiple objects moving non-rigidly using multiple functions.

- Models for the deformation.

\section{References}

1. Chan, T., Vese, L.: Active contours without edges. IEEE Trans. Image Processing 10, 266-277 (2001)

2. Kass, M., Witkin, A., Terzopoulos, D.: Snakes: Active contour models. Int. J. Computer Vision 1, 321-331 (1987)

3. Caselles, V., Kimmel, R., Sapiro, G.: Geodesic active contours. Int. Journal of Computer Vision (1997)

4. Cohen, L.D.: On active contour models and balloons. CVGIP: Image Understanding 53, 211-218 (1991)

5. Xu, C., Prince, J.L.: Snakes, shapes and gradient vector flow. IEEE Trans. on Image Processing 7, 359-369 (1998)

6. Osher, S., Sethian, J.A.: Fronts propagating with curvature-dependent speed: Algorithms based on Hamilton-Jacobi formulations. Journal of Computational Physics 79, 12-49 (1988)

7. Osher, S.J., Fedkiw, R.P.: Level Set Methods and Dynamic Implicit Surfaces. Springer Verlag, Heidelberg (2002)

8. Solem, J.E., Overgaard, N.: A geometric formulation of gradient descent for variational problems with moving surfaces. In: The 5th International Conference on Scale Space and PDE methods in Computer Vision, Scale Space 2005, Hofgeismar, Germany, pp. 419-430. Springer, Heidelberg (2005)

9. Charpiat, G., Keriven, R., Pons, J.P., Faugeras, O.: Designing spatially coherent minimizing flows for variational problems based on active contours. In: ICCV 2005. International Conference on Computer Vision, Beijing, China, pp. 1403-1408. IEEE Computer Society Press, Los Alamitos (2005)

10. Overgaard, N.C., Solem, J.E.: Separating rigid motion for continuous shape evolution. In: International Conference on Pattern Recognition, Hong Kong (2006)

11. Cremers, D., Soatto, S.: Variational space-time motion segmentation. In: International Conference on Computer Vision, Nice, France. vol. 2, pp. 886-892 (2003) 
12. Dervieux, A., Thomasset, F.: A finite element method for the simulation of Rayleigh-Taylor instability. In: Tomas, G., Überhuber, C.W. (eds.) Visualization of Scientific Parallel Programs. LNCS, vol. 771, pp. 145-158. Springer, Heidelberg (1994)

13. Yezzi, A., Mennucci, A.: Conformal metrics and true gradient flows for curves. In: ICCV 2005. International Conference on Computer Vision, Beijing, China, pp. 913-919. IEEE Computer Society Press, Los Alamitos (2005)

14. Chan, T., Zhu, W.: Level set based prior segmentation. Technical Report UCLA CAM Report 03-66, University of California at Los Angeles (2003) 